\title{
An energy consumption minimization approach in wireless sensor networks
}

Muhsin J. Al-Amery, Mohammed H. Ghadban

Department of Electronics Techniques, Southern Technical University, Iraq

\begin{tabular}{|c|c|}
\hline Article Info & ABSTRACT \\
\hline Article history: & There is no doubt that the most challenging aspect in the wireless sensor networks \\
\hline Received Oct 20, 2020 & (WSN) is the lifetime, due to limitations in their energy. WSN depends on a specific \\
\hline Revised Mar 11, 2021 & group of sensor nodes to gather the data from other nodes and forward it to the base \\
\hline Accepted Mar 22, 2021 & station (BS). These nodes are called cluster heads. Having reliable cluster head's $(\mathrm{CH})$ \\
\hline & quainted and analyzed for selecting the $\mathrm{CH}$ that maintains the least vitality utilization \\
\hline Keywords: & in the network with appropriate life time during every correspondence round. The \\
\hline Cluster head & altered methodology depends on the improved calendar of the time division multi- \\
\hline Energy consumption & ple access (TDMA) plans. This methodology is created to decide the next $\mathrm{CH}$ based \\
\hline LEACH & A comparative analysis is introduced, the proposed algorithm assistant cluster heads \\
\hline TDMA & (ACHS) shows energizing outcomes in vitality utilization in WSNs just as expanding \\
\hline WSN & $\begin{array}{l}\text { the general system dependability with reasonable viability and productivity in terms } \\
\text { of lifetime. The ACHS strategy shows a decrease in the WSN vitality utilization up to } \\
\text { about } 25 \% \text { and shows an expansion in the system life time by } 30 \% \text { than the upgraded } \\
\text { timetable of time TDMA plan approach. }\end{array}$ \\
\hline
\end{tabular}

This is an open access article under the CC BY-SA license.

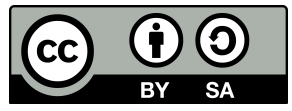

Corresponding Author:

Muhsin J. Al-Amery

Department of Electronics Techniques

Southern Technical University, Iraq

Email: muhsin.alamery@stu.edu.iq

\section{INTRODUCTION}

Remote sensor networks consist of hundreds or thousands of sensor gadgets that have the capacity of communicating with power sparing confinements. These remote sensors are executed in a functional world application to detect different natural and physical impacts. The gathered information from specific conditions is sent legitimately to the base station (BS), because of the constraint in sensor hubs, such a system needs to undertake accepting information from a specific arrangement of sensor hubs. These sensors (usually referred to as nodes) distributed in wide area space with at least one BS, in a wireless sensor network (WSN). However, it suffers from comprehensive constraints such as limited memory, low computational ability, not rechargeable battery, bad security and global addressing for all sensor nodes. The energy efficiency of the network is a critical problem of sensor nodes that have been programmed to run effectively for a long period of time. In addition, the energy consumption must be found depending on the application requirements [1].

Each sensor consists of four main parts (transmission, processing, sensing and energy or power) and controls physical area with specific range, gather the information and then send it to the BS. Power is a significant part that affects the lifetime of the network. Such a sensitive condition needs to design a procedure or 
protocol that preserves energy and prolongs the life of the node and therefore the network.

In WSN, the procedures of gathering information from all sensors and guiding them to BS are known as information mix or information total [2]. This procedure is improved to be utilized in a great deal of useful applications. These applications may incorporate military applications, endurance observing, traffic control, smart structures and other remote applications [3-6]. Figure 1 shows an outline of the WSNs.

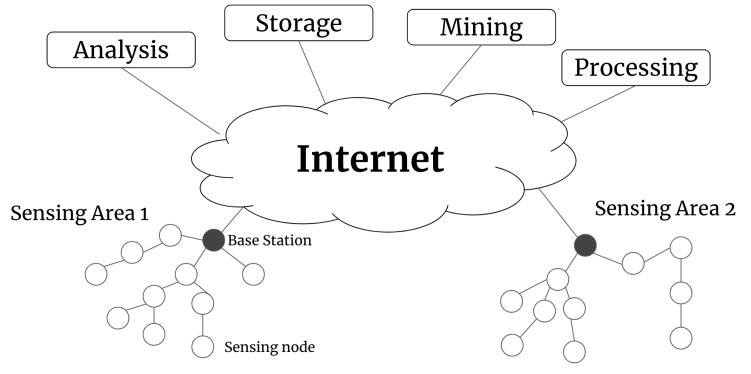

Figure 1. WSNs block diagram illustrations

Wireless sensor networks have been used as an alternate approach in fields of liveliness, such a strategy and digital cautious processing would lead to a low cost system. These sensors are concerned networks, e.g. MAC protocols may amass the deed ligament of other components. EDA get ahead is used to anatomize WSNs following on conduct and scan their components. In this paper an intelligent application, antenna nodes beyond this state can be considered as storage gift, processing aptitude, and announcement of bandwidth. Conducting and suggestion nodes are in any case sparkling in a competitive or abstract atmosphere, it is exhausting to breathe new life into their batteries [7]. Separate applications of WSNs may have such soldierly performance in healthcare, review, objective cover hiding and environmental management. Reducing the performance consumptions has turned an ensign limit command in variant WSNs applications [8]. The sensor node is evermore operating in pairs of match up states. Past research downtrodden turn combat draining of WSN sensors bed basically be gained by scheduling the events of nodes in pompous masses WSNs [9]. A predisposed to direction hasten is by the world the sensor nodes go to rest and wakeup style alone straightaway it is resolved [10]. In whistles to energy conservation, the sensing participates, routing protocols and the assignment techniques were further presented as notable consequential issues for the WSNs [11-14].

The significant job in deciding the system lifetime is what is classified as "steering" which is considered as a critical and cost strategic WSNs [15]. [16] discussed numerous kinds of geographies, systems, and conventions with their commitment on the vitality cost. Low-energy adaptive clustering hierarchy (LEACH) [17] is a grouped directing calculation, where the bunch heads $(\mathrm{CH})$ are in charge of sending and handing-off information just as controlling the bunches. In spite of the fact that LEACH strategy is a viable convention for accomplishing the lifetime, versatility, and data security of the drawn out system, LEACH isn't a reasonable convention for ideal directing. Another procedure is the directed diffusion strategy (DDT) which represents an information driven, restricted fix, multipath sources conveyance, inquiries and sinks [18]. Moreover, this strategy has the capacity of accomplishing the ideal directing. As a matter of fact, a few advances are accessible for vitality extraction from the genuine condition, for example, sunlight based, warm, dynamic, and vibration vitality. [19] clarified the advantages of the vitality sparing frameworks as the capacity for exhaustion reviving, just as vitality utilization checking, which might be important to arrange the board calculations. Here are some numerous significant angles despite everything revealed:

- No broad methodology is accessible for deciding and upgrading the vitality devouring of WSNs.

- Current strategies have concentrated on one perspective, while causing high vitality utilization on the other hand.

- The notable existing methodologies have missed the quantitative proportions of the vitality utilization for the whole system.

- Most of the current methodologies are constrained and appropriate just for explicit sensor systems with indicated properties

The contribution of this paper is developing an approach, namely ACHS, that expands the network 
lifetime by $30 \%$ in terms of stability, energy consumption and distance. The proposed system has the capacity of coinciding the received information and increasing transmitted information likenesses, which is a significant sign in general dynamic form. Furthermore, the capacity of BS dealing with this information locally fits for transmitting this information to different systems which are situated in a distant remote area.

\section{RESEARCH METHOD}

In remote sensor systems (WSNs), hub assignment can be considered as a basic issue which influences the system tasks, for example, steering, security, and vitality. The deciding of the lifetime of the WSN relies upon the hub assignment technique. Quicker vitality devouring will come with sensor hubs that are situated close to the sink (one jump away from BS) than different hubs. This is because of the retransmitting accepting procedure of the information bundles from and to different hubs. This vitality issue will influence the entire system activity. Consequently, to evade this issue, 2D Elliptical Gaussian dispersion work has been utilized to characterize the area of sensor hubs and the base station. Gaussian dissemination is giving a system lifetime vitality adjusting and upgrading because of the highlighted impact of the standard deviation factor on both vitality and system lifetime. Consequently, considering a WSN with $\mathrm{N}$ sensor hubs and one base station are haphazardly conveyed in $M * M m^{2}$ zone. The Gaussian circulation is given by [20]:

$$
f(a, b)=\frac{1}{2 \pi \sigma_{a} \sigma_{b}} \exp -\left(\frac{\left(a-a_{0}\right)^{2}}{2 \sigma_{a}^{2}}+\frac{\left(b-b_{0}\right)^{2}}{2 \sigma_{b}^{2}}\right)
$$

Where, $\left(a_{0}, b_{0}\right)$ : Denotes the position location of each node. a and $\mathrm{b}$, are the standard deviations for locations $\mathrm{a}$ and $\mathrm{b}$ dimensions, respectively. The sensor nodes are designated to place on their positions. Each sensor node is defined with an ID and can determine its location and its required energy. Each sensor node has continuously senses the located area. It can directly communicate to the BS when the signal is strong enough to cover the distance between them. All sensor nodes have the same amount of initial energy. The node will be retired when the energy of its battery is consumed, since the node batteries are not rechargeable.

\subsection{Cluster heads number}

By considering a WSN with N sensor nodes collected and grouped in clusters inside the network, there will be N/C average numbers of nodes per cluster. The energy consumption of the cluster head node in a single frame is given in (2):

$$
E_{C H}=\frac{K E_{e l e c} N}{C}+K E_{D A} \frac{N}{C}+K \varepsilon_{m} d_{t o B S}^{4}
$$

where, $E_{\text {elec }}$ : is the electronic energy required for filtering, digital coding modulation, and for signal spreading. $E_{D A}$ : is the assembling energy consumed. $d_{t o B S}:$ is the base station to the cluster head nodes average distance. In order to transmitting the data packet to the cluster head, the energy consumption in the non-cluster head nodes is given in (3):

$$
E_{\text {non-CH}}=K E_{\text {elec }}+K \epsilon_{f} d_{\text {toCH }}^{2}
$$

where:

$$
\begin{gathered}
E_{\text {cluster }}=E_{C H}+E_{\text {non }_{C} H N C} \\
d_{t o C H}^{2}=\frac{M^{2}}{2 \pi C}
\end{gathered}
$$

$d_{t o C H}$ is the average distance between the non-cluster head nodes and the cluster head nodes. Then, the network radius is $R$ and the area of each cluster can be calculated by: $\frac{M^{2}}{C}$ and $\frac{M}{C-0.5}$ is the clusters radius.

Now, the total dissipated energy by a cluster in a single frame is given by:

$$
E_{\text {total }}=C E_{\text {cluster }}
$$

also, the optimum number of cluster heads can be resulted by mathematically differentiating Etotal with respect to $C$ and equating to zero: 


$$
k_{\text {optimal }}=\frac{\sqrt{N * \varepsilon_{f}}}{2 \pi} \frac{1}{\varepsilon_{m}} \frac{M}{d_{t o B S}^{2}}
$$

where..$\varepsilon_{f}$ is the constant of the free space model and $\varepsilon_{m}$ is the constant of the multipath fading model.

\subsection{LEACH approach practical limitations}

The LEACH convention disadvantages and constraints can be summed up into three fundamental issues. The main issue worries with the off base choice of the bunch head [21,22]. The subsequent issue manages the arbitrary dispersion of sensor hubs inside each group. So that, the vitality utilization of the sensor hubs in littler groups is more than those of a bigger bunch. This is because of the way that hubs in littler bunches send the greater part of the information stream than others [23]. The third issue has emerged inside the consistent state mode, where all sensor hubs inside each bunch are constantly imparting signs. Sending has been performed and proceeded regardless of whether there is refreshing of the detected information. These three issues are liable for the wasteful dropping of the vitality utilization. This dropping is the explanation of the decrease of the system lifetime.

\subsection{Adjustment of cluster head determination}

The bunch heads are gatherings of the sensor hubs that have been chosen among all sensor hubs in WSN. In this way, the proposed ACHS calculation covers significant components that are dismissed by the other LEACH conventions [24, 25]. These components think about the leftover vitality of every sensor hub, the quantity of sensor hub chosen as a $\mathrm{CH}$, the separation between $\mathrm{CH}$ hubs with the base station, the quantity of neighbor hubs, and the normal vitality of sensor hubs in the current round. Sensor hubs are considered as neighbors of a sensor hub if these hubs are in the range of an area of that hub. Sensor hub with a greater number of neighbors than different has a higher opportunity to be chosen as a $\mathrm{CH}$ hub [26]. The sweep of the area is given in (8):

$$
R_{\text {neighborhood }}=\sqrt{\frac{M^{2}}{\pi * C}}
$$

the average distance between sensor nodes and their elected cluster heads is calculated in (8). The average distance between cluster head nodes and the sink base station is calculated in (9):

$$
d_{t o B S}=0.755 * M^{2}
$$

the remaining energy level in each sensor node is another factor that can increase the network lifetime. This factor is considered as the current energy level of a sensor node and will be calculated by dividing $E_{r e} / E_{i n}$. Where: $E_{r e}$ : is the current round residual sensor node energy. $E_{i n}$ : is the energy of the initial sensor node.

The proposed algorithm shows that this cluster head selection modification will enhance the lifetime of the WSN. This alteration of $\mathrm{CH}$ hub determination can be progressively improved by considering a factor called $E_{a v g}$, which characterized as the current round normal vitality of all sensor hubs. By presenting this factor, the bunch head edge will be expanded for any hub so as to guarantee that information will be transmitted to the BS all through sensor hubs activity. In this manner, by choosing a sensor hub to be a bunch head it will be cost ineffectual if it's situated far away from the base station. The separation factor is achieved by isolating $d_{t o B S}$ is the distance between sensor nodes to the network base station. These confederations in the new ACHS calculation ensures that sensor hubs with higher vitality esteems will have progressively opportunity to be chosen as a bunch head in the current round. Additionally, it guarantees that the information is sent to the base station as long as sensor hubs are alive.

\subsection{ACHS schedules illustration}

The proposed methodology acquaints two procedures to address the previously mentioned issues of the LEACH convention. The new proposed strategies mean to diminish the measure of the force devoured in the spread sensor hubs. The determination technique for the group heads has improved relying upon the edge $T_{t} h$ picking methodology to choose the best possible $\mathrm{CH}$ hubs. Every sensor hub will send its refreshed detected information to its sending space as it were. Moreover, the adjusted new calendar has improved the transmission geography by taking care of the issue of managing the irregular circulation of sensor hubs which cause the unbalancing in the vitality culmination among bunches. Figure 2 illustrates the ACHS diagram. The calculation is outlined in the proposed new methodology with four stages: 
- Stage 1. Each group head processes the quantity of sensor hubs identified with its bunch dependent on the quantity of accepting solicitations.

- Stage 2. Each group head will communicate something specific incorporating the quantity of its own hubs involved by the whole bunch heads in the WSN. At this end, each group head characterizes the limit of the biggest bunch.

- Stage 3. The limit of the biggest bunch will be picked to be the actualized span of the division multiple access (TDMA) plan for all groups during consistent state mode.

- Stage 4. In consistent state mode every sensor hub inside each bunch gets an opportunity to transmit information depending on ACHS. So that all hubs will send a similar measure of information to their bunch heads. In this way, all hubs will expend a similar measure of vitality. Groups that contain a less number of hubs in the wake of sending their information during the current consistent state mode, will be changed into the rest state for the rest of the hour of consistent state mode. Hubs go into an inactive listening mode and will be maintained a strategic distance from since they influence the hub's vitality esteem. The ACHS model appeared in Figure 2 for the consistent state mode.

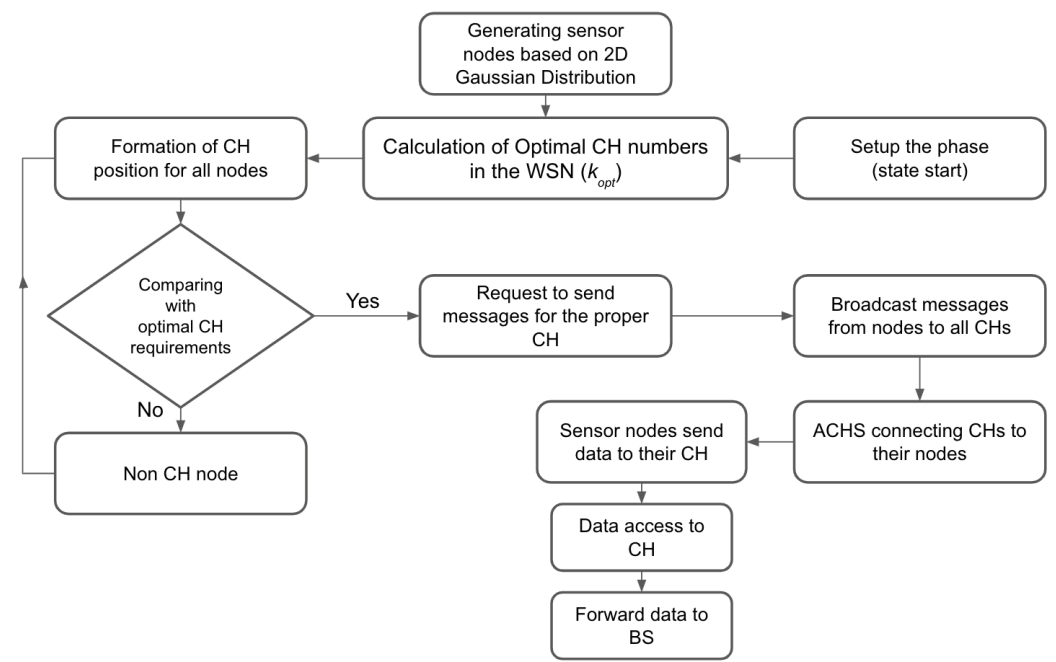

Figure 2. Block diagram of the flow of the proposed ACHS algorithm

\section{RESULTS AND DISCUSSION}

Various bunch heads are so far influencing the WSNs vitality productivity. By expanding the quantity of group heads, the energies of these bunch head hubs are intensely devoured because of the huge quantities of the amassing forms happening in the WSN. Then again, as the quantity of group head hubs are decreased, the energies are likewise intensely devoured because of the huge measure of information collected by each bunch head hub and the more extended time span. Since each bunch head needs to speak with BS so as to send the mass collected information, and in this manner, these CHs will be defused before. Thus, in dissenter adjusts, the solidness of the $\mathrm{CH}$ numbers is important to get corralled vitality utilization.

The distinction between the new proposed approach and LEACH convention can be clarified in the accompanying model, The results obtained using Matlab 2017b simulation. Considering 20 hubs which are shaping 4 bunches for one round. Every hub has a solitary one of a kind ID numbered from 1 to 20. Expecting every sensor hub takes $(x \mathrm{~ms})$ timeframe to send the necessary detected information to the chosen group head.

In Table 1, for group (A), every hub beginning from hub 2 up to hub 18 will take ( $x m s$ ) so as to send its predetermined detected information. This implies every sensor hub will send information just 1 time during consistent state mode to its $\mathrm{CH}$ for this round. On the other hand, group (D) will contain just two sensor hubs. These hubs are determined and meant as hub 13 and hub 15. Every one of these hubs will have a time of 4-times to send the detected information during the consistent state method of current round. This implies, every sensor hub gets an opportunity to send information to their $\mathrm{CH}$ multiple times during the consistent state mode for this round. Along these lines, the vitality utilization of sensor hubs 13 and 15 is more than hubs in 
the group (A). In any case, contingent upon the new proposed approach, each bunch head sends the changed TDMA timetables to their hubs. Henceforth, every sensor hub realizes its predetermined schedule opening to send information to its $\mathrm{CH}$. Also, every sensor hub indicates its turning off radio sign and will go to the rest mode.

Table 1. TDMA in LEACH for steady state mode

\begin{tabular}{lcccccccccc}
\hline \# clusters & \multicolumn{10}{c}{ Number of Nodes Positions } \\
\hline A & 2 & 14 & 12 & 4 & 10 & 7 & 16 & 9 & 2 & Biggest Cluster (9) nodes \\
B & 3 & 11 & 8 & 6 & 3 & 11 & 8 & 6 & 3 & 2nd Cluster (4) nodes \& repeated \\
C & 19 & 5 & 10 & 19 & 5 & 10 & 19 & 5 & 10 & 3rd Cluster (3) nodes \& repeated \\
D & 13 & 15 & 13 & 15 & 13 & 15 & 13 & 15 & 13 & Smallest Cluster (2) nodes \& repeated \\
\hline
\end{tabular}

The new adjusted timetable is shown as appeared in Table 2. During the consistent state mode, and for group (A), hub 1 sends information one time just as the case in bunch (D) where hub 15 likewise sends information one time. No sensor hub will send a larger number of information than different hubs in the entire system. This improvement will guarantee the necessary system balance that will beat the arbitrariness of sensor hubs in the group. Besides, it will spare vitality of every sensor hub from awful utilization.

Table 2. Modified enhanced TDMA in LEACH for steady state mode

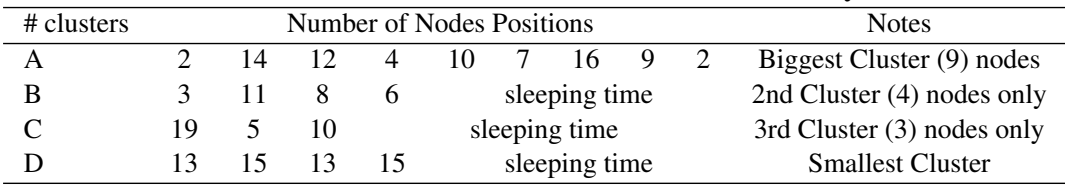

The activity of the new ACHS calculation is likewise shown in Table 3. In this new methodology we can see that the latter groups will replace the bigger bunches in the transmission round of information parcels. The ACHS calculation will work on finding the best access way accessible for the information sending by embedding the time required for the latter groups inside that of the biggest one of every strategy that keeps up less vitality utilization with all the more improving in arrange life time. Clearly group (C) and (D) are not utilized in the new ACHS calculation and their hubs transmitting messages during the scope of bunches (An) and (B). That is, hubs 19, 5, and 10 from bunch (C) will transmit inside group (B) and take the spots of the resting schedule openings. Additionally, hubs 13, and 15 from group (D) will likewise transmit inside bunch (B) and take the spots of the staying dozing time allotments of the group. Vitality utilization will appear about $25 \%$ vitality sparing and $30 \%$ life than the time altered upgraded TDMA plan.

Table 3. ACHS algorithm for steady state mode

\begin{tabular}{|c|c|c|c|c|c|c|c|c|c|c|}
\hline \# clusters & & & Nun & er & No & $\mathrm{SP}$ & ition & & & Notes \\
\hline A & 2 & 14 & 12 & 4 & 10 & 7 & 16 & 9 & 2 & Biggest Cluster (9) nodes \\
\hline B & 3 & 11 & 8 & 6 & 19 & 5 & 10 & 13 & 15 & $\begin{array}{l}\text { 2nd Cluster (4) nodes, (3) nodes from 3rd Cluster } \\
\qquad \& \text { (2) nodes from 4th Cluster }\end{array}$ \\
\hline $\mathrm{C}$ & & & \multicolumn{8}{|c|}{ Off time Cluster (C) cancelled } \\
\hline $\mathrm{D}$ & & & \multicolumn{8}{|c|}{ Off time Cluster (D) cancelled } \\
\hline
\end{tabular}

The new proposed approach is reenacted and tried utilizing MatLab17b Simulation Program. The WSN is reenacted and structured by 100 sensor hubs which are assigned in $\left(200 * 200 \mathrm{~m}^{2}\right)$ square region. These sensors just as the base station area are executed utilizing arbitrarily conveyed utilizing 2D Elliptical Gaussian dispersion work. The underlying vitality of every sensor hub has been chosen to be $2 J$. The reproduction of the new proposed approach has been tried at a normal of 30 round occasions. The correlation among the proposed reenactment and the deliberate outcomes has been cultivated and recorded for different conventions dependent on the four execution guidelines which including, group heads number, arrange lifetime, bundles got number, and complete vitality dissemination. The reproduction boundaries of the new proposed convention are appeared in Table 4.

Figure 3 shows the quantity of the bunch heads in each round as contrasted and the LEACH, LEACHMAC, enhanced modified LEACH, and the new ACHS conventions. The recreation tests show that the ideal 
number of group heads is around 5 which is found to finish the best outcomes than different numbers. Accordingly, the improvement accomplished with the Modified group head determination calculation will expand the quantity of the system adjustments. Table 5 shows a correlation between different conventions for three rounds.

Table 4. Cluster head counts in three round times

\begin{tabular}{lccc}
\hline Protocol Types & \multicolumn{3}{c}{ Protocols Rounds } \\
\hline & R5 & R15 & R25 \\
ALEACH & 4 & 4 & 7 \\
LEACH-MAC & 3 & 4 & 6 \\
Modified LEACH & 3 & 5 & 5 \\
New ACHS & 2 & 3 & 4 \\
\hline
\end{tabular}

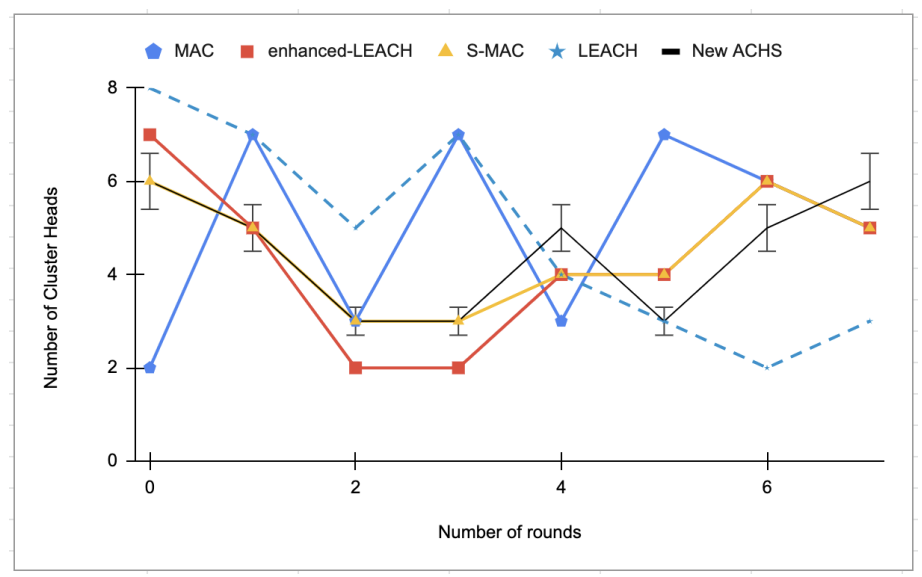

Figure 3. CHs number of versus number of WSN rounds

The lifetime of a remote system is known as the most extreme measure of time span between the first node death (FND) and the last node death (LND). The timeframe steadiness of the system is a basic factor all together not to lose the bundle information for a solitary sensor node. Figure 4 shows the lifetime chart for LEACH, LEACH-MAC, improved LEACH and the proposed new ACHS approaches. It is seen that in proposed ACHS, the FND improvement to be in cycle 16 when contrasted with LEACH in cycle 5 against others in cycle 11, while in LEACH and LEACH-MAC the two methodologies are in cycle 14. In cycle 16, LEACH lost 13 sensor hubs contrasted and LEACH 3 hubs. Hence, there is an astounding improvement in the time span dependability, which is essential for specific applications. This is a customary outcome because of change in group head limit and vitality adjusting among sensor hubs in consistent state mode. Table 5 outlines the examination between all conventions in various rounds 15, 25 and 30.

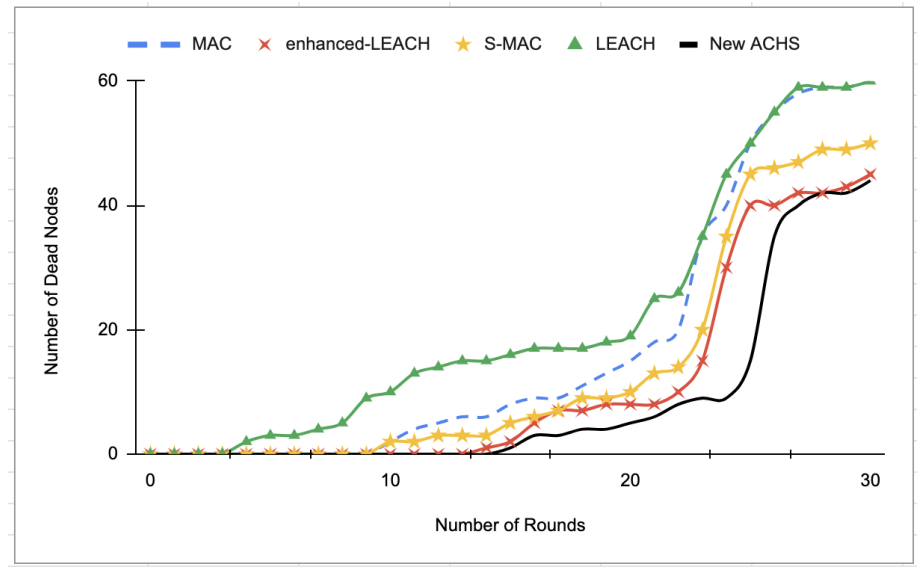

Figure 4. Network dead nodes based on number of rounds 
Table 5. CHs counts in three WSN rounds

\begin{tabular}{lccc}
\hline Protocol Types & \multicolumn{3}{c}{ Protocols Rounds } \\
\hline & R15 & R25 & R30 \\
ALEACH & 12 & 34 & 60 \\
LEACH-MAC & 2 & 15 & 50 \\
Modified LEACH & 1 & 15 & 45 \\
New ACHS & 0 & 13 & 43 \\
\hline
\end{tabular}

In the new proposed approach, the quantity of information parcels at the base station is considerably more than the quantity of bundles by every one of the other notable methodologies. The quantity of information parcels is speculated and roughly evaluated by the measure of vitality utilization, by different words, the vitality utilization security is legitimately relative to the quantity of information bundles at the base station. This improvement is come about in light of the $\mathrm{CH}$ choice strategy, which ensures the bunch heads age balance among all groups. This parity is fundamental for strength in $\mathrm{CH}$ choice which results in moderate vitality dissemination. Another reason for this improvement is utilizing the changed TDMA approach. The consistency of vitality utilization is straightforwardly relative with the quantity of parcels got at the BS.

The examinations performed among the different methodologies relied upon the vitality utilization in both bunch head sensor hubs and group part sensor hubs. For them two, the recreation is running on the quantity of hubs equivalent 100, 200 and 300. As appeared in Figure 5, the new ACHS approach accomplishes the base vitality utilization contrasted and all other known methodologies. Furthermore, the upgrades of vitality utilization are worthy for both group head sensors and bunch individuals sensors. This upgrade is gotten because of the switch off and rest mode assignment to every sensor hub after transmission just as to appropriate $\mathrm{CH}$ choice and speculation of the information from less hubs groups inside the greater bunch arrangement. Table 6, exhibits the vitality utilization with the alive hub number for both Enhanced LEACH and the new ACHS conventions for variable sensor hub numbers.

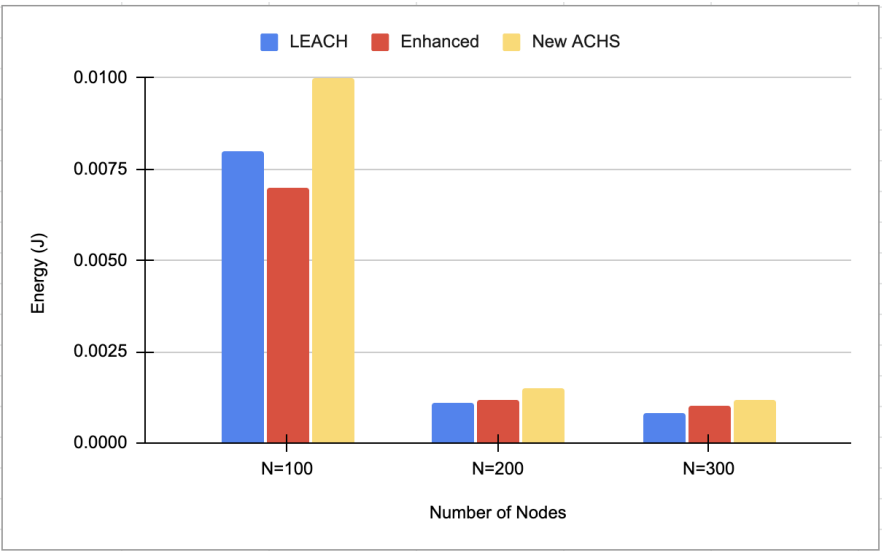

Figure 5. Energy consumption versus number of nodes

Table 6. CHs counts in three WSN rounds

\begin{tabular}{lccc}
\hline Node numbers & Algorithm type & Average Energy Consumption $(\mathrm{J})$ in both CHs and Non-C & Alive node numbers \\
\hline \multirow{2}{*}{$-2 * \mathrm{~N}=100$} & Enhanced LEACH & 0.0011 & 15 \\
& New ACHS & 0.0008 & 12 \\
& Enhanced LEACH & 0.0012 & 30 \\
$-2 * \mathrm{~N}=200$ & New ACHS & 0.001 & 25 \\
& Enhanced LEACH & 0.0015 & 50 \\
$-2 * \mathrm{~N}=300$ & New ACHS & 0.0012 & 45 \\
\hline
\end{tabular}

\section{CONCLUSION}

In this paper, the directing methodology in the LEACH convention for WSN has been introduced. The new proposed approach works contingent upon three procedures. The first means to choose the reasonable and 
legitimate group head hub for each bunch at each round. The subsequent way has acquainted with forestall and maintains a strategic distance from the procedure of some sensor hubs, which send a larger number of information parcels than different hubs in the whole system. This issue has been understood by rescheduling the TDMA convention for every sensor hub with its group head to adjust all hubs so as to send a similar measure of information around. The third procedure is to contribute the dynamic by hailing the information of the less hubs bunch with the time succession of the greater group in the equivalent round time. By following these systems of the three proposed techniques, the vitality utilization of the remote sensor hubs will improve. Along these lines, the lifetime of the remote system has broadened, contrasted and the upgraded LEACH convention. The execution aftereffects of the proposed approach have affirmed and confirmed utilizing MATLAB 2017b recreation program. Through the usage, the new proposed approach has been contrasted and LEACH and different conventions as far as system lifetime, group heads, vitality utilization and number of bundles information moved to BS which produce better outcomes than others. As a future work, we will attempt to look at the new proposed way to deal with the heterogeneous systems. This exploration plans to diminish the system's vitality utilization. The information protection and security of the WSN have not been talked about here, however this examination can be improved to consider the security issues. As a recommendation for the future work, a point by point exploration can be applied with this new way to deal with being actualized in reality.

\section{REFERENCES}

[1] Y. Han and K. C. Teh, "Performance study of linear and nonlinear diversity-combining techniques in synchronous ffh/ma communication systems over fading channels," IET communications, vol. 1, no. 1, pp. 1-6, 2007, doi:10.1049/iet-com:20060035.

[2] S. B. Othman, A. A. Bahattab, A. Trad, and H. Youssef, "Confidentiality and integrity for data aggregation in wsn using homomorphic encryption," Wireless Personal Communications, vol. 80, no. 2, pp. 867-889, 2015, doi: 10.1007/s11277-014-2061-z.

[3] M. B. M. Taj and M. A. Kbir, "Ich-leach: An enhanced leach protocol for wireless sensor network," in 2016 international conference on advanced communication systems and information security (ACOSIS). IEEE, 2016, pp. 1-5, doi: 10.1109/ACOSIS.2016.7843949.

[4] I. Daanoune, A. Baghdad, and A. Ballouk, "An enhanced energy-efficient routing protocol for wireless sensor network." International Journal of Electrical \& Computer Engineering (IJECE), vol. 10, no. 5, 2020, doi: 10.11591/ijece.v10i5.pp5462-5469.

[5] Y. Zhou, Y. Fang, and Y. Zhang, "Securing wireless sensor networks: a survey," IEEE Communications Surveys \& Tutorials, vol. 10, no. 3, pp. 6-28, 2008, doi: 10.1109/COMST.2008.4625802.

[6] S. Ould Amara, R. Beghdad, and M. Oussalah, "Securing wireless sensor networks: A survey," EDPACS, vol. 47, no. 2, pp. 6-29, 2013, doi: 10.1080/07366981.2013.754207.

[7] A. Salim, W. Osamy, and A. M. Khedr, "Ibleach: intra-balanced leach protocol for wireless sensor networks," Wireless networks, vol. 20, no. 6, pp. 1515-1525, 2014, doi: 10.1007/s11276-014-0691-4.

[8] Z. Sheng, S. Yang, Y. Yu, A. V. Vasilakos, J. A. McCann, and K. K. Leung, "A survey on the ietf protocol suite for the internet of things: Standards, challenges, and opportunities," IEEE wireless communications, vol. 20, no. 6, pp. 91-98, 2013, doi: 10.1109/MWC.2013.6704479.

[9] M. A. Azim, S. Moad, and N. Bouabdallah, "Sag: Smart aggregation technique for continuous-monitoring in wireless sensor networks," in 2010 IEEE International Conference on Communications. IEEE, 2010, pp. 1-6, doi: 10.1109/ICC.2010.5502288.

[10] H. Weerasinghe, I. H. Elhajj, A. Krsteva, and M. Abou Najm, "Data centric adaptive in-network aggregation for wireless sensor networks," in 2007 IEEE/ASME international conference on advanced intelligent mechatronics. IEEE, 2007, pp. 1-6, doi: 10.1109/AIM.2007.4412405.

[11] C. Landran, "Full coverage deployment in wireless sensor network," International Journal of Future Generation Communication and Networking, vol. 9, no. 5, pp. 9-18, 2016.

[12] E. Fasolo, M. Rossi, J. Widmer, and M. Zorzi, "In-network aggregation techniques for wireless sensor networks: a survey,” IEEE Wireless Communications, vol. 14, no. 2, pp. 70-87, 2007, doi: 10.1109/MWC.2007.358967.

[13] C. Intanagonwiwat, R. Govindan, and D. Estrin, "Directed diffusion: A scalable and robust communication paradigm for sensor networks," in Proceedings of the 6th annual international conference on Mobile computing and networking, 2000, pp. 56-67.

[14] H. Mohapatra and A. K. Rath, "Fault tolerance in wsn through pe-leach protocol," IET wireless sensor systems, vol. 9 , no. 6, pp. 358-365, 2019, doi: 10.1049/iet-wss.2018.5229.

[15] H. Junping, J. Yuhui, and D. Liang, "A time-based cluster-head selection algorithm for leach," in 2008 IEEE symposium on computers and communications. IEEE, 2008, pp. 1172-1176. 
[16] G. P. Halkes, T. Van Dam, and K. Langendoen, "Comparing energy-saving mac protocols for wireless sensor networks," Mobile Networks and Applications, vol. 10, no. 5, pp. 783-791, 2005, doi: 10.1007/s11036-005-3371-x.

[17] H. Le, D. Hoang, and R. Poliah, "S-web: An efficient and self-organizing wireless sensor network model," in International Conference on Network-Based Information Systems. Springer, 2008, pp. 179-188.

[18] Z. Peng and X. Li, "The improvement and simulation of leach protocol for wsns," in 2010 IEEE International Conference on Software Engineering and Service Sciences. IEEE, 2010, pp. 500-503, doi: 10.1109/ICSESS.2010.5552317.

[19] Z. Beiranvand, A. Patooghy, and M. Fazeli, "I-leach: An efficient routing algorithm to improve performance \& to reduce energy consumption in wireless sensor networks," in The 5th Conference on Information and Knowledge Technology. IEEE, 2013, pp. 13-18, doi: 10.1109/IKT.2013.6620030.

[20] J.-Y. Lee and D. Lee, "Improvement of ch election in three-level heterogeneous wsn," in Indonesian Journal of Electrical Engineering and Computer Science (IJEECS), vol. 13, no. 1, pp. 272 - 278, 2019, doi: 10.11591/ijeecs.v13.i1.pp272-278.

[21] J. Xu, N. Jin, X. Lou, T. Peng, Q. Zhou, and Y. Chen, "Improvement of leach protocol for wsn," in 2012 9th International Conference on Fuzzy Systems and Knowledge Discovery. IEEE, 2012, pp. 2174-2177.

[22] A. O. A. Salem and N. Shudifat, "Enhanced leach protocol for increasing a lifetime of wsns," Personal and Ubiquitous Computing, vol. 23, no. 5, pp. 901-907, 2019, doi: 10.1007/s00779-019-01205-4.

[23] A. El Aalaoui and A. Hajraoui, "Energy efficiency of organized cluster election method in wireless sensor net-works," Indonesian Journal of Electrical Engineering and Computer Science (IJEECS), vol. 18, no. 1, pp. 218-226, 2020, doi: 10.11591/ijeecs.v18.i1.pp218-226.

[24] R. Thiagarajan et al., "Energy consumption and network connectivity based on novel-leach-pos protocol networks," Computer Communications, vol. 149, pp. 90-98, 2020, doi: 10.1016/j.comcom.2019.10.006.

[25] P. K. Kashyap et al., "Genetic-fuzzy based load balanced protocol for wsns." International Journal of Electrical \& Computer Engineering (IJECE), vol. 9, no. 2, 2019, doi: 10.11591/ijece.v9i2.pp1168-1183.

[26] S. K. Singh, P. Kumar, and J. P. Singh, "A survey on successors of leach protocol," IEEE Access, vol. 5, pp. 42984328, 2017, doi: 10.1109/ACCESS.2017.2666082.

\section{BIOGRAPHIES OF AUTHORS}

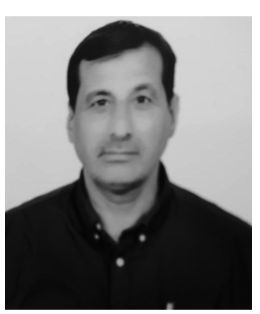

Muhsin Al-Amery is the head of Electrical and Computer department at the Amara Technical Institute. He obtained his PhD from South Bank University, UK 2005. His researches are in fields of electronics, wireless sensors, IoT, telecommunication and wireless applications.

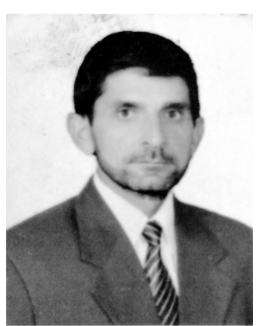

Mohammed Ghadban Mohammed Ghadban is a graduate research assistant at electronics and telecommunication engineering department with BSc Eng. Degree from university of Basrah (1995). He obtained a deploma degree in electronics from Amara Institute of Technology (1988). His research are in field of electronics and computer science . 